\title{
Thermodynamic Concept for Quantification of Water-Energy-Food Nexus
}

\author{
Julia Stoyanova* \\ National Institute of Meteorology and Hydrology, Bulgaria
}

Submission: April 03, 2020; Published: April 09, 2020

*Corresponding author: Julia Stoyanova, National Institute of Meteorology and Hydrology, Tzarigradsko schosse 66, 1784 Sofia, Bulgaria

\section{Abstract}

A thermodynamic approach for water-energy-food nexus quantification is presented, and its application is demonstrated. Appliyng irreversible thermodynamics of steady-state to the boundary layer between the ecosystem canopy and environment, the entropy production in the main photosynthetic apparatus is quantified. This quantity is used as an intergal quantitative criteria for efficiency of ecosystem functioning. The role of entropy production as a measure for conversion of solar energy in biochemical energy (growth substances) at the specific site environment (microclimate, soil, and topography), species and phenology is motivated. Applying these thermodynamic concept and methods analysis to agrosystems of winter wheat is performed. Analytical description of the accumulated entropy production and crop yield is obtained. Applicability of satellite information from Meteosat in entropy production quantification and yield prediction is demonstrated.

Keywords: Thermodynamics of steady-state; Modeling water-energy-food nexus; Entropy production; Satellite-based land surface temperature; Yield; Agro-ecosystem

\section{Introduction}

Growth, development and productivity of ecosystems are strongly dependent on their functional coupling with the environment via biogeophysical and biogeochemical functional relations and feedback mechanisms. Carbon cycle is fundamental to life on Earth and it determines the amount of carbon accumulated in yield. Its spatial and temporal dynamics interplay at different environmental conditions requires quantification of water-energy-food nexus (WEFN). The basic question that should be answered in this case is: how to quantify the efficiency of ecosystem functioning, which in the case of agricultural practice is reflected by food production?

To answer this, a thermodynamic concept dealing with the energetic nature of the main life processes going in ecosystems, and their quantitative description is presented in this study. It is applied for quantification of WEFN and corresponding yield production referring to agricultural practice. Considering that ecosystems are functioning as open thermodynamic systems far from steady-state, the main challenge is how to apply the first and the second laws of thermodynamics for WEFN reliable description? An adequate quantitative description of the energetic aspects of WEFN is expected to give answer how can be quantified the functional relation between the environment and yield production. Considering that food production is a problem of global concern, the second important consideration in this work is to propose a methodology for application of contemporary satellite technology (in particular satellite information from Meteosat) as an alternative source of data for description of climate and food relations.

\section{The Concept}

Applying the linear irreversible thermodynamics of steady-state according to the surface-boundary approach in biothermodynamics [1,2], and accounting for the functional causeconsequence link between biogeophysical and biogeochemical cycles $[3,4]$, the energetic aspects of water-energy-food nexus in ecosystems are quantitatively described. The standpoint of this thermodynamic approach is the universal law of Rubner (1883) and its energetic interpretation in describing themain physiological processes in an ecosystem. According to the Rubner's law, at equal environmental conditions the rate of specific metabolism increases with the decrease of organisms' size, along with the increase of their specific surface (where: the specific metabolism is defined as the rate of metabolism per a unit volume or weight of the organism; the specific surface is the proportion between the organisms' surface to its volume). The surface law of Rubner that 
is valid for whole vivid world, terrestrial- as well as aquatic- plant species [5] provokes two basic problems in description of their functioning.

\section{What is the reason for the functional relationship between the rate of specific metabolism and the specific surface of an organism?}

Since the metabolism is an energetic process its quantitative description might be realized using the laws of thermodynamics. Analyses [1] reveal that the rate of specific metabolism of organisms is not an individual biological characteristic but appears to be biophysically determined feature by the processes going on the boundary surface between the organism and environment. Otherwise, energetic conversions at a molecular level, i.e. metabolic processes are regulated by mass- and energyexchanges at the surface boundary organism-environment. That means, considering the law of Rubner, the thermodynamic forces and fluxes should be identified at the boundary surface organismenvironment. Such an approach of studying the main processes in living organisms is introduced as "the surface-boundary approach in biothermodynamics" [1]. A thermodynamic analysis of multicomponent system with a variable composition, which is functioning at constant environmental temperature and pressure, has revealed the main thermodynamic forces and fluxes acting at the boundary surface. These fluxes are identified to be the waterand heat-exchange provoked by gradients of temperature and chemical potential of water at the boundary surface.

The next problem to be solved is how to apply thermodynamic laws to ecosystems, who are functioning far from steady-state. The answer is that the mass- and energy- exchange rates might be identified by using linear irreversible thermodynamics [6] because the linearity between the fluxes and correspond forces (gradients) at the surface-boundary layer organism-environment [7] is preserved. The applicability of the linear irreversible thermodynamics means that energetic conversions at molecular level, i.e. metabolic processes might be quantified by mass and energy exchange processes at the surface boundary organism (in particular leaf system of ecosystems)-environment, namely because of applicability of phenomenological equations of Onsager (linking the main fluxes and driving gradients). In other words, the cause for the metabolic rate of an organism depends on the rate of main thermodynamic fluxes acting at a unit of the boundary surface.

\section{Which is the mechanism (in a thermodynamic sense) of converting an environmental stimulus to metabolic process that in turn modifies the quantity and quality of growth?}

Because the anisotropic nature of living membranes, the "forbidden" of Curie is not valid and the transport processes through them (like vectors) can be coupled with the metabolic reactions (like scalars), [7]. For example, water dynamics through an organism as provoked by gradient at the boundary surface leaf system-environment could be coupled with the biochemical reactions, and to be quantitatively described by the phenomenological equations of Onsager, using irreversible thermodynamics of steady-state. Consequently, the application of linear irreversible thermodynamics to the boundary layer ecosystems leave system-environment indicates that conversion of the Gibbs free energy of moving water (like vector) through an anisotropic media might be coupled with biochemical reactions (like scalars) at molecular level, and thus to be considered as a universal energetic mechanism for heterotrophic type of metabolism.

\section{Coupling between biogeophysical and biogeochemical cycles}

The energy conversions at a molecular level (biochemical processes) are the basis for growth in a plant organism. In accordance to the first law of thermodynamics, the biosynthesis of growth substances requires an inner source of energy, which is the respiratory substrate, respectively the dark respiration. For quantification of specific ecosystem-site capacity to utilize the solar energy into biochemical energy and related growth response, the second law of irreversible thermodynamics has to be applied. The thermodynamic description of heterotrophic type metabolic processes as regulated by mass- and energy exchange at the surface boundary organism-environment $[1,7]$ implies the analytical description of both: the conversion rate of free Gibbs energy of moving (transpiring) water into biochemical one and the efficiency of this conversion at different environment. In other words, assessment of energy relations between dark transpiration and respiration considering the energy losses during this conversion process is required.

The difference in the rate of processes going at the boundary leaves-environment (biogeophysical cycling) has been used to regulate the contents of important for growth metabolites (organic phosphorus, nucleic acids, ascorbic acid and others) and yield production [4]. Consequently, it is approved that the intensity of biogeophysical cycle appears to be the cause for the efficiency of biogeochemical cycle as a consequence $[3,4]$. Functional coupling between biogeophysical and biogeochemical cycles is the bases for crop yield production. Biogeophysical cycle is the signature of functioning of the ecosystems as open thermodynamic systems through mass- and energy- exchange at the specific site environment (soil, climate, topography, etc.).

The energetic loses accompanying ecosystem functioning are quantified through the entropy production (dS/dt, characterized by function of dissipation $\Phi$ ) in the main photosynthetic apparatus, the leaf system. $\Phi$ is proposed as a complex thermodynamic criteria for characterizing bioenergetic climatic resources, and quantified as a sum of the entropy produced during the heat- and during the water- exchange between leaves and environment (Eq. (1), [1,2,7]. $\Phi$ is a dynamic biophysical characteristics of ecosystems and can reflect the environmental (meteorological- 
climate) resources and vegetation capacity to accumulate solar energy in biochemical energy (responsible for growth and yield production).

$$
\Phi=T \frac{\Delta_{i} S}{\Delta t}=\frac{H \Delta T}{T \Delta t}+L E \frac{\Delta q}{\Delta t}
$$

$\Delta S$ - entropy production in leaves; E - evapotranspiration rate; $\mathrm{H}$ - leaf-air heat exchange rate; $\mathrm{T}$ - mean leaf-air absolute temperature; $\Delta \mathrm{T}$ - leaf-air temperature difference; $\mathrm{q}_{\mathrm{s}}$ - specific humidity of saturated water vapour at the leaf surface at corresponding leaf temperature; $\Delta \mathrm{q}=\left(\mathrm{q}_{\mathrm{s}}-\mathrm{q}\right)$ - the difference between specific air humidity at the transpiring leaf level and this of surrounding air, $\mathrm{L}$ - latent heat of vaporization of water.

\section{Application}

The Surface-boundary thermodynamic approach [1,2] has been applied for energetic description of processes in single leaves, whole plant organisms and forest phytocoenoses.

The applicability of the thermodynamic concept is here illustrated by quantification of the entropy production $\Phi$ in canopy leaves of agroecosystem of winter wheat according Eq. (1) and it's linking to corresponding yield production. Two experimental sites Dobrich $(43.57 \mathrm{~N}, 27.83 \mathrm{E})$ and Kneza (43.5N, 24.08E) from the agrometeorological network of the National Institute of Meteorology and Hydrology (NIMH) are selected. They are located at different microclimate environment, south-eastern Europe, Bulgaria. Accumulated $\Phi$ during the period, critical for wheat yield formation is calculated for each year within the period (2007-2018). Results are presented on Figure 1a, and indicate that winter wheat field in the region of Dobrich (red line) is characterized by a lower value of dissipated energy $\Phi$, i.e. more energy is involved in growth substances. This trend is consistent for all 12 years of the test (with a single exception for 2009). (a)

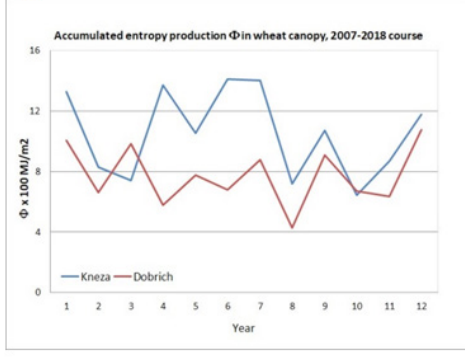

(b)

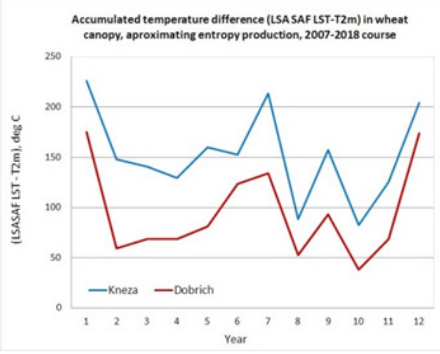

(c)

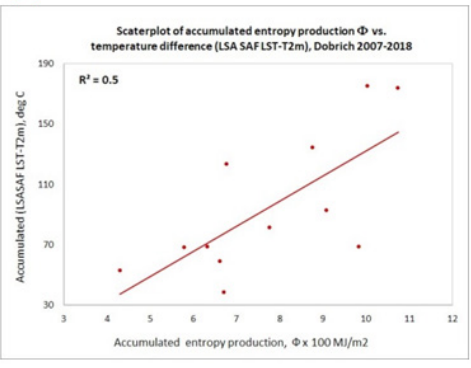

Figure 1: Thermodynamic entropy production (characterized by dissipation function, $\Phi$ ) accumulated during the critical for yield formation period of wheat ecosystems. Dataset: 2007-2018 years, two experimental sites Dobrich (red line), Kneza (blue line): a) $\Phi$ calculation according Eq. 1, running by parameters of meteorological measurements; b) $\Phi$ approximated by (LSASAF LST-T2m) temperature difference as a first proxy of heat exchange component, Eq. 1, using Meteosat information; c) Scatterplot for $\Phi$ derived by the two approaches a) and b).

While in this case the model is run using as inputs meteorological parameters from measurements from operational NIMH network, its applicability is also illustrated by using information from geostationary Meteosat satellites. The use of satellite data for analyzing and predicting climate resources and related food production is of increased importance due to the societal vulnerability to climate variability and change. The problem of using satellite technology to advance our understanding of complicated energy-water-carbon nexus to produce food has two main aspects. First to apply a relevant physical description that secondly allows transferring this knowledge for operational application accounting for space-time variability as seen by satellites [8].

In this relation, $\Phi$ is approximated by the temperature difference between the skin temperature (as retrieved by the LSASAF Land Surface Temperature, LST product) and air temperature at $2 \mathrm{~m}$ height $(\mathrm{T} 2 \mathrm{~m})$. The (LSASAF LST-T2m) 
temperature difference is applied as a first proxy of the heat flux exchange between the wheat canopy and atmosphere. Results (Figure 1b) illustrate that the trend in entropy production derived using satellite information is similar as derived by modeling (Figure 1a). Wheat field in the region of Dobrich has a lower heat exchange component (LSASAF LST-T2m) used as a proxy of $\Phi$ for each of year from the test period.

Finally, $\Phi$ calculations by modelling (run from parameters from measurements) and run by using available Meteosat Secong Generation (MSG) satellites information are compared, Figure 1c. There is a good agreement between the entropy productions derived by the two methods; a high correlation, $\mathrm{R}^{2}=0.5$ for both experimental sites (here is presented only the scaterrplot for Dobrich region) is observed. Following the presented thermodynamic view of ecosystem functioning it is illustrated that data from meteorological geostationary satellites are useful for characterizing bioenergetic climatic resources, which are responsible for carbon accumulation and yield production.

Following the presented thermodynamic concept, $\Phi$ is used to be linked with the crop yield production. Measurements for wheat yield from the two experimental sites of the NIMH agrometeorological network are used and compared with the accumulated (accounting for the critical for wheat yield formation phenological period) $\Phi$ values (Figure 2). The increase of energetic looses characterized by $\Phi$ leads to a decrease of wheat yield, analytically described by a 2-rank polynomial dependance. There is a high correlation between $\Phi$ and yield production for both microclimate environment, $\mathrm{R}^{2}$ ranging between 0.5 and 0.6 . For the region of Kneza (Figure 2a) $\Phi$ is quantifies after Eq. 1, using meteorological measurements to run the model; for the region of Dobrich (Figure 2b) $\Phi$ is quantifies after Eq. 1, using (LSASAF LST$\mathrm{T} 2 \mathrm{~m}$ ) temperature difference. (a)

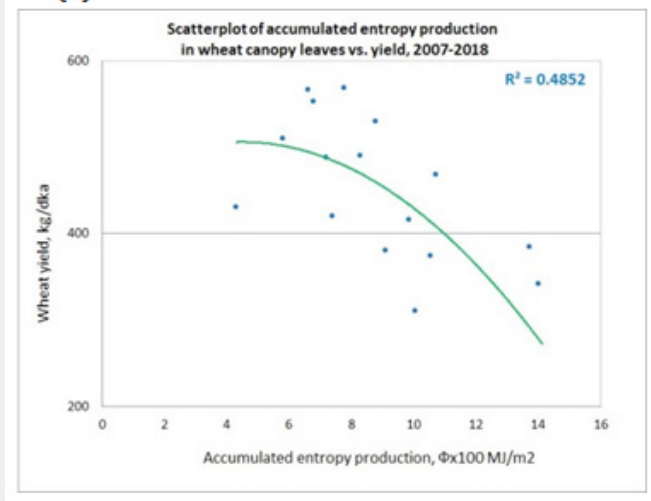

(b)

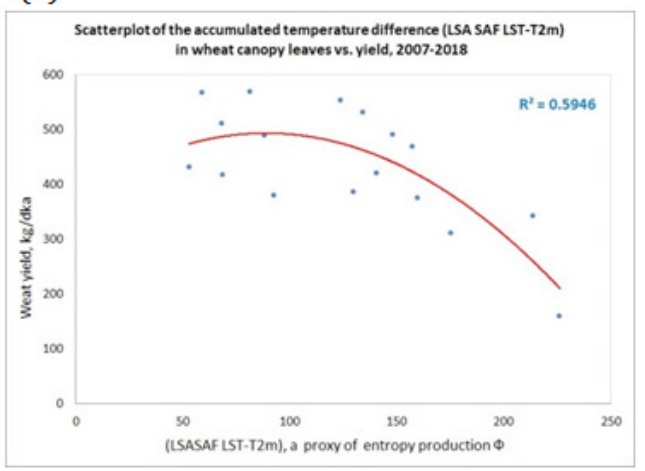

Figure 2: Analytical description of the accumulated entropy production in wheat canopy leaves vs. yield: a) $\Phi$ (Kneza) quantified after Eq. 1 , using meteorological measurements; b) $\Phi$ (Dobrich) quantified after Eq. 1, using satellite information (LSASAF LST-T2m). Dataset: 20072018; accumulation performed for the phenological period critical for wheat yield formation.

\section{Concluding Remarks}

A conceptual framework for water-energy-carbon nexus quantification, based on thermodynamic description of energy conversions during ecosystem functioning is described. Applying irreversible thermodynamics of steady state, the efficiency of ecosystem functioning in terms of yield production is characterized by quantification of the accompanying energetic loses. The energetic loses are quantified by the thermodynamic entropy production, $\Phi$ and a model of its calculation is proposed. The concept adds to the understanding of agroecosystems sustainable functioning and the yield potential of grain crops under optimum growing conditions (without weeds, pests and deceases). Based on this interdisciplinary approach, $\Phi$ is introduced to serve as a measure of specific ecosystem-site capacity to utilize the solar energy into biochemical energy and related growth response/ crop yield. The proposed index integrates a complex set of soil-wheatatmosphere data and processes into a single quantity that can be further evaluated for different climate and soil environment. Its value would assists in decision making for agroclimatic resources and how climate change may affect them.

Regarding global forecasting applications, in-situ observations cannot fulfill alone the modeling needs. It is shown that satellites data can be used in our thermodynamic model for diagnoses/ forecast of agricultural system functioning. It is illustrated that this modeling approach is applicable at any other region using satellite information (in this case from Meteosat) for accounting specific crop, climate, soil environment and $\Phi$ calculation. Remote sensing techniques based on Earth Observation data products have a potential for assessing the impact environmental change on agricultural production. 


\section{Acknowledgement}

The research was partially sponsored by (EUMETSAT SALGEE Project) with grant number (PO Number/Date 4500017699/22-January-2019) and the fee for publication is payed by the EUMETSAT LSASAF CDO3 Project.

\section{References}

1. Florov RI (1988) Thermodynamics of biosystems. Surface-boundary approach in biothermodynamics. Sofia, p. 158.

2. Florov RI (2002) Surface-Boundary Approach in Biothermodynamics and its Applications. Journal of Shenyang Institute of Chemical Technology 16(4): 241-249.

3. Stoyanova JS (1996) Relationship between transpiration and respiration in plants during the dark period. Biologia plantarum 38 77-83.
4. Stoyanova JS (1998) A Thermodynamic Insight into the Relationship: Dark Transpiration, Respiration, and Growth in Plants. In: Tsekos I, Moustakas M (Eds.), Progress in Botanical Research, Kluwer Acad Publ, Springer, USA, pp. 234-246.

5. Odum EP (1961) Fundamentals of ecology. ( $2^{\text {nd }}$ edn), Published 1961 by W. B. Saunders Company, Philadelphia, p. 546.

6. Denbig KG (1958) The Thermodynamics of the Steady State. Methuen \& Co., Ltd, ASIN: B001N45K6Q p. 118.

7. Florov RI (1983) Rubner's law and applicability of linear (Onager's) thermodynamics of irreversible processes to living systems. Zh Obsh Biol (Moskva) 44: 305-315.

8. Stoyanova JS, Georgiev CG (2019) Thermodynamic View of WaterEnergy-Carbon Nexus in the Context of Satellite Data Application for Food Security2019 Joint EUMETSAT/AMS/NOAA Satellite Conference, 29 September - 4 October 2019 in Boston, MA.

\section{Your next submission with Juniper Publishers} will reach you the below assets

- Quality Editorial service

- Swift Peer Review

- Reprints availability

- E-prints Service

- Manuscript Podcast for convenient understanding

- Global attainment for your research

- Manuscript accessibility in different formats ( Pdf, E-pub, Full Text, Audio)

- Unceasing customer service

Track the below URL for one-step submission https://juniperpublishers.com/online-submission.php 